\title{
The Height of a Class in the Cohomology Ring of Polygon Spaces
}

\author{
Yasuhiko Kamiyama and Kazufumi Kimoto \\ Department of Mathematics, University of the Ryukyus, Nishihara-Cho, Okinawa 903-0213, Japan \\ Correspondence should be addressed to Yasuhiko Kamiyama; kamiyama@sci.u-ryukyu.ac.jp
}

Received 26 September 2013; Accepted 9 December 2013

Academic Editor: Hernando Quevedo

Copyright (c) 2013 Y. Kamiyama and K. Kimoto. This is an open access article distributed under the Creative Commons Attribution License, which permits unrestricted use, distribution, and reproduction in any medium, provided the original work is properly cited.

Let $\bar{M}_{n, r}$ be the configuration space of planar $n$-gons having side lengths $1, \ldots, 1$ and $r$ modulo isometry group. For generic $r$, the cohomology ring $H^{*}\left(\bar{M}_{n, r} ; \mathbb{Z}_{2}\right)$ has a form $H^{*}\left(\bar{M}_{n, r} ; \mathbb{Z}_{2}\right)=\mathbb{Z}_{2}\left[R(n, r), V_{1}, \ldots, V_{n-1}\right] / \mathscr{I}_{n, r}$, where $R(n, r)$ is the first Stiefel-Whitney class of a certain regular 2-cover $\pi: M_{n, r} \longrightarrow \bar{M}_{n, r}$ and the ideal $\mathscr{I}_{n, r}$ is in general big. For generic $r$, we determine the number $h(n, r)$ such that $R(n, r)^{h(n, r)} \neq 0$ but $R(n, r)^{h(n, r)+1}=0$.

\section{Introduction}

Given a string $\mathbf{r}=\left(r_{1}, \ldots, r_{n}\right)$ of $n$ positive real numbers, one considers the configuration space $\bar{M}_{\mathrm{r}}$ of planar polygon linkages having side lengths $r_{i}$ modulo isometry group. Starting in [1-3], the topology of $\bar{M}_{\mathbf{r}}$ has been considered by many authors. A notable achievement is [4] which determined the mod 2 cohomology ring $H^{*}\left(\bar{M}_{\mathbf{r}} ; \mathbb{Z}_{2}\right)$ for generic $\mathbf{r}$. The study culminated in the proof by [5] of a conjecture by Kevin Walker which states that one can recover relative lengths of edges from the mod 2 cohomology ring of the configuration space.

By [4], the cohomology ring $H^{*}\left(\bar{M}_{\mathbf{r}} ; \mathbb{Z}_{2}\right)$ has a form

$$
H^{*}\left(\bar{M}_{\mathbf{r}} ; \mathbb{Z}_{2}\right)=\frac{\mathbb{Z}_{2}\left[R_{\mathbf{r}}, V_{1}, \ldots, V_{n-1}\right]}{\mathscr{I}_{\mathbf{r}}},
$$

where $R_{\mathbf{r}}$ is the first Stiefel-Whitney class of a certain regular 2-cover $\pi: M_{\mathbf{r}} \rightarrow \bar{M}_{\mathbf{r}}$ and the ideal $\mathscr{I}_{\mathbf{r}}$ is in general big. For example, when $\mathbf{r}=(1, \ldots, 1), \mathscr{I}_{\mathbf{r}}$ is generated by polynomials whose number is approximately $2^{n-2}$. While [5] made clever arguments to distinguish the cohomology rings, it is difficult to extract more concrete information from the ring. The reason is that the polynomials do not form a Gröbner basis.

In this direction, one natural problem is to compute the height of $R_{\mathbf{r}}$, that is, the unique $h(\mathbf{r})$ such that $R_{\mathbf{r}}^{h(\mathbf{r})} \neq 0$ but $R_{\mathbf{r}}^{h(\mathbf{r})+1}=0$. The purpose of this paper is to determine $h(\mathbf{r})$ for the case $\mathbf{r}=(1, \ldots, 1, r)$. Note that such $\mathbf{r}$ is a string which comes next to the equilateral case. For example, the homology groups $H_{*}\left(M_{\mathbf{r}} ; \mathbb{Z}\right)$ were determined in [6].

Finally, we note that the height of an element in the cohomology ring of a space $X$ has been studied in order to give nice lower bounds on the Lusternik-Schnirelmann category of $X$. For example, [7] studied the problem for the case that $X=G_{k}\left(\mathbb{R}^{n+k}\right)$ and the element is the first StiefelWhitney class of the universal bundle.

This paper is organized as follows. In Section 2 we state our main results. Theorem A determines the height of $R_{\mathbf{r}}$. Theorem $\mathrm{B}$ determines which element represents the mod 2 fundamental class of $\bar{M}_{\mathbf{r}}$. In Section 3 we prove auxiliary results. In Section 4 we prove Theorem $B$ and in Section 5 we prove Theorem A.

\section{Main Results}

For $4 \leq n \in \mathbb{N}$ and $0<r \in \mathbb{R}$, we define the configuration space of planar $n$-gons having side lengths $1, \ldots, 1$ and $r$ by

$$
M_{n, r}=\left\{\left(z_{1}, \ldots, z_{n-1}\right) \in\left(S^{1}\right)^{n-1} \mid \sum_{i=1}^{n-1} z_{i}=r\right\} .
$$

Here $z_{i} \in S^{1} \subset \mathbb{C}$ denotes the unit vectors in the direction of the sides of a polygon. Note that $M_{n}$ comes with a natural involution

$$
\tau: M_{n, r} \longrightarrow M_{n, r}, \quad \tau\left(z_{1}, \ldots, z_{n-1}\right)=\left(\bar{z}_{1}, \ldots, \bar{z}_{n-1}\right) .
$$


We set

$$
\bar{M}_{n, r}=\frac{M_{n, r}}{\tau} .
$$

It is clear that $\bar{M}_{n, r}=\emptyset$ if $r>n-1$ and $\bar{M}_{n, n-1}=$ one point $\}$. In [1-3] it is proved that if $r$ is a natural number which satisfies $r \leq n-2$ and has the same parity as $n$, then there is a diffeomorphism

$$
\bar{M}_{n, r^{\prime}} \cong \bar{M}_{n, r}, \quad \forall r^{\prime} \in(r-1, r+1) .
$$

Moreover, if $n$ is even and $r^{\prime} \in(0,1)$, then we have $\bar{M}_{n, r^{\prime}} \cong$ $M_{n-1,1} \times{ }_{\tau} S^{1}$, where $\tau$ acts on $S^{1}$ by complex conjugate. On the other hand, if $n>4$ and $r \in \mathbb{N}$ has the different parity as $n$, then $\bar{M}_{n, r}$ has singular points.

Hereafter we assume that $r$ is a natural number which satisfies $r \leq n-2$ and has the same parity as $n$. In this case, $\bar{M}_{n, r}$ is a connected closed manifold of dimension $n-3$. Moreover, $\bar{M}_{n, r}$ is orientable if and only if $n$ is even.

The following examples are well known:

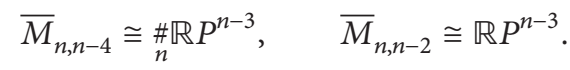

The following theorem is crucial in this paper.

Theorem 1 (see [4, Corollary 9.2]). The mod 2 cohomology ring of $\bar{M}_{n, r}$ is

$$
H^{*}\left(\bar{M}_{n, r} ; \mathbb{Z}_{2}\right)=\frac{\mathbb{Z}_{2}\left[R(n, r), V_{1}, \ldots, V_{n-1}\right]}{\mathscr{I}_{n, r}},
$$

where $R(n, r)$ and $V_{1}, \ldots, V_{n-1}$ are of degree 1 and $\mathscr{I}_{n, r}$ is the ideal generated by the three families of elements:

(R1)

$$
V_{i}^{2}+R(n, r) V_{i}, \quad \text { where } i=1, \ldots, n-1,
$$

$\prod_{i \in S} V_{i}, \quad$ where $S \subset\{1, \ldots, n-1\}$ is such that $|S| \geq \frac{n-r}{2}$,

(R3)

$$
\begin{aligned}
& \sum_{S \subset L} R(n, r)^{|L-S|-1} \prod_{i \in S} V_{i}, \text { where } \\
& L \subset\{1, \ldots, n-1\} \text { is such that }|L| \geq \frac{n+r}{2} .
\end{aligned}
$$

The symbol $S$ in $(R 3)$ runs over all subsets of L including the empty set. By (R2) a term of the sum in (R3) vanishes if $|S| \geq(n-r) / 2$.

The class $R(n, r)$ coincides with the first Stiefel-Whitney class of the regular 2-cover $\pi: M_{n, r} \rightarrow \bar{M}_{n, r}$. We define the height of $R(n, r)$ is the unique $h(n, r)$ such that $R(n, r)^{h(n, r)} \neq 0$ but $R(n, r)^{h(n, r)+1}=0$.
In order to state our main results, we prepare notations. Throughout this paper, the notation $a \equiv b$ means that $a \equiv$ $b(\bmod 2)$. We set

$$
\begin{gathered}
D(n)=n-2, \quad e(n, r)=\frac{n-r}{2}-1, \\
k(n, r)=\max \{i \mid 0 \leq i \leq e(n, r)-1, \\
\left.\left(\begin{array}{c}
D(n)-e(n, r)+i \\
i
\end{array}\right) \equiv 1\right\} .
\end{gathered}
$$

Moreover, we set

$$
\phi(n, r)=\left\{\begin{array}{lr}
n-3, & \text { if }\left(\begin{array}{c}
D(n) \\
e(n, r)
\end{array}\right) \equiv 1, \\
\frac{n+r}{2}+k(n, r)-2, & \text { if }\left(\begin{array}{c}
D(n) \\
e(n, r)
\end{array}\right) \equiv 0 .
\end{array}\right.
$$

Now our first result is the following.

Theorem A. For all $n \geq 4$ and $r \in \mathbb{N}$ which satisfies $r \leq n-2$ and has the same parity as $n$, are has $h(n, r)=\phi(n, r)$. if

We study the generator of $H^{n-3}\left(\bar{M}_{n, r} ; \mathbb{Z}_{2}\right)=\mathbb{Z}_{2}$. Note that

$$
1 \leq v_{1}<\cdots<v_{i} \leq n-1
$$

then we have in $H^{n-3}\left(\bar{M}_{n, r} ; \mathbb{Z}_{2}\right)$ that

$$
R(n, r)^{n-3-i} V_{1} \cdots V_{i}=R(n, r)^{n-3-i} V_{v_{1}} \cdots V_{v_{i}} .
$$

We set

$$
p(n, r, i)=R(n, r)^{n-3-i} V_{1} \cdots V_{i} .
$$

Our second result is the following.

Theorem B. Let $n$ and $r$ be as in Theorem A. Then for $0 \leq i \leq$ $e(n, r)$, the following equality holds in $H^{n-3}\left(\bar{M}_{n, r} ; \mathbb{Z}_{2}\right)$ :

$$
p(n, r, i)=\left(\begin{array}{c}
D(n)-i \\
e(n, r)-i
\end{array}\right)
$$

Theorem A implies that $R(n, r)^{n-3}$ is a generator if and only if $\left(\begin{array}{c}D(n) \\ e(n, r)\end{array}\right) \equiv 1$. This is in agreement with Theorem B for $i=0$.

For the case of almost equilateral polygons, that is, the case for $r=1$ or 2 , we can write Theorem A more explicitly.

Proposition C. (i) About $\bar{M}_{n, 1}$ for oddn, one has the following.

(a) One has $\left(\begin{array}{c}D(n) \\ e(n, r)\end{array}\right) \equiv 1$ if and only if $n$ is of the form $n=$ $2^{s}+1$.

(b) If $n$ satisfies that

$$
2^{s}+1 \leq n \leq 2^{s+1}-1,
$$

then $h(n, 1)=2^{s}-2$. 
(c) One has $p\left(2^{s}+1,1, i\right) \neq 0$ for all $i$.

(ii) About $\bar{M}_{n, 2}$ for even $n$, one has the following.

(a) One has $\left(\begin{array}{c}D(n) \\ e(n, r)\end{array}\right) \equiv 1$ if and only if $n$ is of the form $n=$ $2^{s}$.

(b) If $n=2^{s}$, then $h(n, 2)=2^{s}-3$. On the other hand, if $n$ satisfies that

$$
2^{s}+2 \leq n \leq 2^{s+1}-2,
$$

then $h(n, 2)=2^{s}-2$.

(c) One has $p\left(2^{s}, 2, i\right) \neq 0$ for all $i$.

One deduces the proposition from Theorem A in Section 5 .

We give two examples of Theorem A. The first example is about the case when $n$ is small.

Example 2. We consider Table 1 for the case $h(n, r)=n-$ 3. Then $p(n, r, i)=0$ (where $p(n, r, i)$ is defined in (15)) in $H^{n-3}\left(\bar{M}_{n, r} ; \mathbb{Z}_{2}\right)$ if and only if $(n, r, i)$ satisfies the case which is given in Table 2 .

Our second example is about the case when $r$ is large.

Example 3. (i) We have

$$
h(n, n-4)= \begin{cases}n-3 & n \text { is odd } \\ n-4 & n \text { is even }\end{cases}
$$

(ii) We have $h(n, n-2)=n-3$.

In fact, (i) for odd $n$ and (ii) correspond to the case $\left(\begin{array}{c}D(n) \\ e(n, r)\end{array}\right) \equiv 1$ in Theorem A. But we know a stronger result as in (6).

\section{Auxiliary Results}

Proposition 4. Let $x$ be indeterminate and $e \in \mathbb{N}$. Let $A_{e}(x)$ be an e $\times$ e matrix over $\mathbb{Z}_{2}[x]$ defined as follows:

$$
A_{e}(x)=\left(\left(\begin{array}{c}
x+i \\
j
\end{array}\right)\right)_{\substack{1 \leq i \leq e \\
1 \leq j \leq e}} .
$$

Then one has the following results.

(i) One has rank $A_{e}(x) \geq e-1$.

(ii) One has rank $A_{e}(x)=e$ if and only if $\left(\begin{array}{c}x+e \\ e\end{array}\right) \neq 0$ in $\mathbb{Z}_{2}[x]$.

(iii) When rank $A_{e}(x)=e-1$, the following results hold.

(a) Let ${ }^{t} \mathbf{y}={ }^{t}\left(y_{1}, \ldots, y_{e}\right)$ be the unique nontrivial element of $\operatorname{Ker} A_{e}(x)$. Then one has

$$
y_{j}=\left(\begin{array}{c}
x+e-j \\
e-j
\end{array}\right) \text {. }
$$

(b) Let $^{t} \mathbf{y}={ }^{t}\left(y_{1}, \ldots, y_{e}\right)$ be as in (a). Then one has

$$
\sum_{j=1}^{e}\left(\begin{array}{c}
x+e+1 \\
j
\end{array}\right) y_{j}=1 .
$$

Proof. We define 4 matrices as follows:

$$
\begin{aligned}
& P_{e}=\left(\left(\begin{array}{c}
i \\
j
\end{array}\right)\right)_{\substack{1 \leq i \leq e \\
0 \leq j \leq e}}, \quad Q_{e}(x)=\left(\left(\begin{array}{c}
x \\
j-i
\end{array}\right)\right)_{\substack{0 \leq i \leq e \\
1 \leq j \leq e}}, \\
& \widehat{P}_{e}=\left(\left(\begin{array}{l}
i \\
j
\end{array}\right)\right)_{1 \leq i, j \leq e}, \quad \widehat{Q}_{e}(x)=\left(\left(\begin{array}{c}
x \\
j-i
\end{array}\right)\right)_{1 \leq i, j \leq e} .
\end{aligned}
$$

Since det $\widehat{P}_{e}=\operatorname{det} \widehat{Q}_{e}(x)=1$, they are regular matrices.

We write $P_{e}$ and $Q_{e}(x)$ as follows:

$$
P_{e}=\left(\begin{array}{ll}
1 & \widehat{P}_{e}
\end{array}\right), \quad Q_{e}(x)=\left(\begin{array}{c}
\mathbf{q} \\
\widehat{Q}_{e}(x)
\end{array}\right)
$$

Using the Chu-Vandermonde identity

$$
\left(\begin{array}{c}
x+i \\
j
\end{array}\right)=\sum_{k=0}^{j}\left(\begin{array}{l}
i \\
k
\end{array}\right)\left(\begin{array}{c}
x \\
j-k
\end{array}\right),
$$

we have

$$
A_{e}(x)=P_{e} Q_{e}(x)=\mathbf{1 q}+\widehat{P}_{e} \widehat{Q}_{e}(x) .
$$

(i) Since rank $\mathbf{1 q}=1$ and $\widehat{P}_{e} \widehat{Q}_{e}(x)$ is a regular matrix, we have rank $A_{e}(x) \geq e-1$.

(ii) Note that

$$
\operatorname{det} A_{e}(x)=\left(\begin{array}{c}
x+e \\
e
\end{array}\right)
$$

In fact, both the sides coincide for $x=0$ and vanish for $x \in \mathbb{Z}$ such that $-e \leq x \leq-1$.

Now (27) tells us that $\operatorname{rank} A_{e}(x)=e$ if and only if $\left(\begin{array}{c}x+e \\ e\end{array}\right) \neq 0$.

(iii)(a) Since $y_{e}=1$, we have ${ }^{t} \mathbf{y} \neq 0=$. We must prove that

$$
\sum_{j=1}^{e}\left(\begin{array}{c}
x+i \\
j
\end{array}\right)\left(\begin{array}{c}
x+e-j \\
e-j
\end{array}\right)=0
$$

for $1 \leq i \leq e$. Since we are assuming that rank $A_{e}(x)=e-1$, it will suffice to prove that

$$
\sum_{j=0}^{e}\left(\begin{array}{c}
x+i \\
j
\end{array}\right)\left(\begin{array}{c}
x+e-j \\
x
\end{array}\right)=0 .
$$

We use the formula

$$
\sum_{j=0}^{c}(-1)^{j}\left(\begin{array}{l}
a \\
j
\end{array}\right)\left(\begin{array}{l}
b-j \\
b-c
\end{array}\right)=\left(\begin{array}{c}
b-a \\
c
\end{array}\right)
$$

which holds over $\mathbb{Z}$. Setting $a=x+i, b=x+e$, and $c=e$, we have in $\mathbb{Z}_{2}$ that the left-hand side of (29) is $\left(\begin{array}{c}e-i \\ e\end{array}\right)$. Since $1 \leq i \leq e$, this is 0 .

(b) The left-hand side of (b) is that of (29) for $i=e+1$. Then this is $\left(\begin{array}{c}e-i \\ e\end{array}\right)=\left(\begin{array}{c}-1 \\ e\end{array}\right)=1$. 
TABLE $1: h(n, r)$ for $4 \leq n \leq 20$.

\begin{tabular}{lllllllllllllllllll}
\hline$r$ & & & & & & & & \multicolumn{1}{c}{$n$} & & & & & & & \\
& 4 & 5 & 6 & 7 & 8 & 9 & 10 & 11 & 12 & 13 & 14 & 15 & 16 & 17 & 18 & 19 & 20 \\
\hline 1 & - & 2 & - & 2 & - & 6 & - & 6 & - & 6 & - & 6 & - & 14 & - & 14 & - \\
2 & 1 & - & 2 & - & 5 & - & 6 & - & 6 & - & 6 & - & 13 & - & 14 & - & 14 \\
3 & - & 2 & - & 4 & - & 6 & - & 6 & - & 6 & - & 12 & - & 14 & - & 14 & - \\
4 & - & - & 3 & - & 4 & - & 6 & - & 6 & - & 11 & - & 12 & - & 14 & - & 14 \\
5 & - & - & - & 4 & - & 6 & - & 6 & - & 10 & - & 12 & - & 14 & - & 14 & - \\
6 & - & - & - & - & 5 & - & 6 & - & 9 & - & 10 & - & 13 & - & 14 & - & 14 \\
7 & - & - & - & - & - & 6 & - & 8 & - & 10 & - & 10 & - & 14 & - & 14 & - \\
8 & - & - & - & - & - & - & 7 & - & 8 & - & 10 & - & 10 & - & 14 & - & 14 \\
9 & - & - & - & - & - & - & - & 8 & - & 10 & - & 10 & - & 14 & - & 14 & - \\
10 & - & - & - & - & - & - & - & - & 9 & - & 10 & - & 13 & - & 14 & - & 14 \\
11 & - & - & - & - & - & - & - & - & - & 10 & - & 12 & - & 14 & - & 14 & - \\
12 & - & - & - & - & - & - & - & - & - & - & 11 & - & 12 & - & 14 & - & 14 \\
13 & - & - & - & - & - & - & - & - & - & - & - & 12 & - & 14 & - & 14 & - \\
14 & - & - & - & - & - & - & - & - & - & - & - & - & 13 & - & 14 & - & 17 \\
15 & - & - & - & - & - & - & - & - & - & - & - & - & - & 14 & - & 16 & - \\
16 & - & - & - & - & - & - & - & - & - & - & - & - & - & - & 15 & - & 16 \\
17 & - & - & - & - & - & - & - & - & - & - & - & - & - & - & - & 16 & - \\
18 & - & - & - & - & - & - & - & - & - & - & - & - & - & - & - & - & 17 \\
\hline
\end{tabular}

TABLE 2: The cases for $p(n, r, i)=0$.

\begin{tabular}{lc}
\hline$(n, r)$ & $i$ \\
\hline$(9,3)$ & 1 \\
$(13,7)$ & 1 \\
$(15,5)$ & 1,3 \\
$(17,3)$ & $1,3,5$ \\
$(17,5)$ & 2,3 \\
$(17,7)$ & $1,2,3$ \\
$(17,11)$ & 1 \\
\hline
\end{tabular}

Corollary 5. Assume that $\operatorname{rank} A_{e}(x)=e-1$ and let ${ }^{t} \mathbf{y}$ be the element in Proposition 4(iii)(a). One sets

$$
\ell(x, e)=\min \left\{j \mid 1 \leq j \leq e, y_{j} \equiv 1\right\}
$$

If one has also that $\operatorname{rank} A_{e}(x-1)=e-1$, then one has

$$
\ell(x-1, e)=\ell(x, e)-1
$$

Proof. The unique nontrivial element of $\operatorname{Ker} A_{e}(x-1)$ is given by

$$
{ }^{t}\left(y_{1}+y_{2}, y_{2}+y_{3}, \ldots, y_{e-1}+y_{e}, y_{e}\right)
$$

The corollary is clear from this.

The following property of $\phi(n, r)$ will be used in Section 5.
Lemma 6. (i) If $r \geq 2$ and $\left(\begin{array}{c}D(n) \\ e(n, r)\end{array}\right) \equiv 0$, one has

$$
\phi(n-1, r-1)=\phi(n, r) .
$$

(ii) If $n$ is odd and satisfies that

$$
2^{s}+1 \leq n \leq 2^{s+1}-1
$$

then the following results hold.

(a) One has $\left(\begin{array}{c}D(n) \\ e(n, r)\end{array}\right) \equiv 1$ if and only if $n$ is of the form $n=$ $2^{s}+1$

(b) $\phi(n, 1)=2^{s}-2$.

Proof. (i) We need to consider two cases according to $\left(\begin{array}{c}D(n-1) \\ e(n-1, r-1)\end{array}\right) \equiv 0$ or 1 .

Case $(\mathrm{a})\left(\left(\begin{array}{c}D(n-1) \\ e(n-1, r-1)\end{array}\right) \equiv 0\right)$.

Note that

$$
\begin{gathered}
D(n-1)=D(n)-1=n-3, \\
e(n-1, r-1)=e(n, r)=\frac{n-r}{2}-1 .
\end{gathered}
$$

We shall prove that

$$
k(n-1, r-1)=k(n, r)+1 .
$$

We consider Proposition 4 for $x=D(n)-e(n, r)$ and $e=$ $e(n, r)$. For $\mathbf{y}$ in the proposition, we set $\tilde{y}_{j}=y_{e-j}$ and put $\widetilde{\mathbf{y}}=\left(\widetilde{y}_{0}, \ldots, \widetilde{y}_{e-1}\right)$. Note that $\widetilde{\mathbf{y}}$ is the sequence which appears in the definition of $k(n, r)$. This and the definition of $\ell(x, e)$ in Corollary 5 tell us that

$$
k(n, r)=e(n, r)-\ell(D(n)-e(n, r), e(n, r)) .
$$


Using Corollary 5, (36), and (37), we have

$$
\begin{aligned}
& \ell(D(n-1)-e(n-1, r-1), e(n-1, r-1)) \\
& \quad=\ell(D(n)-e(n, r), e(n, r))-1 .
\end{aligned}
$$

Combining (37), (39), and (40), we obtain (38).

Now (i) follows from the following computation:

$$
\begin{aligned}
\phi(n-1, r-1) & =\frac{n-1+r-1}{2}+(k(n, r)+1)-2 \\
& =\phi(n, r) .
\end{aligned}
$$

Case (b) $\left(\left(\begin{array}{c}D(n-1) \\ e(n-1, r-1)\end{array}\right)\right) \equiv 1$.

By definition, we have $\phi(n-1, r-1)=n-4$. Hence it will suffice to prove $\phi(n, r)=n-4$. This is equivalent to the assertion that $k(n, r)=e(n, r)-1$ and also equivalent to the assertion that $\left(\begin{array}{c}D(n)-1 \\ e(n, r)-1\end{array}\right) \equiv 1$. But the last assertion is clear if we apply (36) and (37) to our assumptions that

$$
\left(\begin{array}{c}
D(n) \\
e(n, r)
\end{array}\right) \equiv 0, \quad\left(\begin{array}{c}
D(n-1) \\
e(n-1, r-1)
\end{array}\right) \equiv 1 .
$$

(ii) If we prove (b), then (a) is clear from this. Since (b) clearly holds for $n=2^{s}+1$, we assume that $2^{s}+3 \leq n \leq 2^{s+1}-1$. We set $n=2 m+1$. By definition,

$$
k(n, r)=\max \left\{i \mid 0 \leq i \leq m-2,\left(\begin{array}{c}
m+i \\
i
\end{array}\right) \equiv 1\right\} .
$$

By Kummer, $\left(\begin{array}{c}a+b \\ b\end{array}\right)$ is odd if and only if there are no carries when adding $a$ and $b$ in base 2 . Hence if we write $m=2^{s-1}+u$ (where $1 \leq u \leq 2^{s-1}$ ), the greatest $i$ (where $i \leq m-2$ ) such that $\left(\begin{array}{c}m+i \\ i\end{array}\right) \equiv 1$ is

$$
i=2^{s-2}+2^{s-3}+\cdots+1-u=2^{s-1}-1-u .
$$

Hence $k(n, r)=2^{s-1}-1-u$ and

$$
\begin{aligned}
\phi(n, 1) & =(m+1)+k(n, r)-2 \\
& =\left(2^{s-1}+u+1\right)+\left(2^{s-1}-1-u\right)-2=2^{s}-2 .
\end{aligned}
$$

Modifying Proposition 4 slightly, we have the following.

Lemma 7. Let $\check{A}_{e}(x)$ be an $\mathrm{e} \times(e+1)$ matrix over $\mathbb{Z}_{2}[x]$ defined as follows:

$$
\check{A}_{e}(x)=\left(\begin{array}{c}
x+i \\
j
\end{array}\right)_{\substack{1 \leq i \leq e \\
0 \leq j \leq e}} .
$$

Then one has the following results.

(i) One has $\operatorname{rank} \check{A}_{e}(x)=e$.

(ii) Let ${ }^{t} \check{\mathbf{y}}={ }^{t}\left(\check{y}_{0}, \ldots, \check{y}_{e}\right)$ be the unique nontrivial element of $\operatorname{Ker} \breve{A}_{e}(x)$. Then one has

$$
\check{y}_{j}=\left(\begin{array}{c}
x+e-j \\
e-j
\end{array}\right)
$$

Proof. (i) Let $P_{e}$ be the matrix in the proof of Proposition 4 and we define

$$
\check{Q}_{e}(x)=\left(\left(\begin{array}{c}
x \\
j-i
\end{array}\right)\right)_{\substack{0 \leq i \leq e \\
0 \leq j \leq e}}
$$

Since $\check{A}_{e}(x)=P_{e} \breve{Q}_{e}(x)$, rank $P_{e}=e$, and det $\breve{Q}_{e}(x)=1$, the result follows;

(ii) follows from (29).

\section{Proof of Theorem B}

For $(n+r) / 2 \leq i \leq n-2$, let $f_{i}\left(R(n, r), V_{1}, \ldots, V_{n-1}\right)$ be the polynomial in Theorem 1 (R3) for $L=\{1, \ldots, i\}$. Note that the coefficient of $p(n, r, j)$ in

$$
R(n, r)^{n-2-i} f_{i}\left(R(n, r), V_{1}, \ldots, V_{n-1}\right)
$$

is $\left(\begin{array}{l}i \\ j\end{array}\right)$, where $p(n, r, j)$ is defined in (15). We list the coefficient in the following $e(n, r) \times(e(n, r)+1)$ matrix $B_{n, r}$ :

$$
B_{n, r}=\left(\left(\begin{array}{l}
i \\
j
\end{array}\right)\right)_{\substack{((n+r) / 2) \leq i \leq n-2 \\
0 \leq j \leq e(n, r)}} .
$$

Theorem 1 tells us that

$$
\begin{aligned}
B_{n, r}{ }^{t} & (p(n, r, 0), \ldots, p(n, r, j), \ldots, p(n, r, e(n, r))) \\
& ={ }^{t}(0, \ldots, 0, \ldots, 0) .
\end{aligned}
$$

In the notation of Lemma 7, we have

$$
B_{n, r}=\check{A}_{e(n, r)}\left(\frac{n+r}{2}-1\right) \text {. }
$$

Since $p(n, r, j)(0 \leq j \leq e(n, r))$ generate $H^{n-3}\left(\bar{M}_{n, r} ; \mathbb{Z}_{2}\right),(51)$ tells us that

$$
p(n, r, j)=\check{y}_{j}
$$

We compute $\check{y}_{j}$ as follows:

$$
\begin{aligned}
\check{y}_{j} & =\left(\begin{array}{c}
\left(\frac{n+r}{2}-1\right)+\left(\frac{n-r}{2}-1\right)-j \\
e(n, r)-j
\end{array}\right) \\
& =\left(\begin{array}{c}
n-2-j \\
e(n, r)-j
\end{array}\right)=\left(\begin{array}{c}
D(n)-j \\
e(n, r)-j
\end{array}\right) .
\end{aligned}
$$

\section{Proof of Theorem A}

Theorem $\mathrm{A}$ is proved by showing the upper bound for those $\lambda$ with $R(n, r)^{\lambda} \neq 0$ coinciding with the lower bound. About the upper bound, we have the following.

Proposition 8. In $H^{*}\left(\bar{M}_{n, r} ; \mathbb{Z}_{2}\right)$, one has $R(n, r)^{\phi(n, r)+1}=0$. Proof. Since $\operatorname{dim} \bar{M}_{n, r}=n-3$, the case for $\left(\begin{array}{c}D(n) \\ e(n, r)\end{array}\right) \equiv 1$ is clear. Hence we may assume that $\left(\begin{array}{c}D(n) \\ e(n, r)\end{array}\right) \equiv 0$. For $p$ in 
$((n+r) / 2) \leq p \leq n-2$, we denote by $g_{p}\left(R(n, r), V_{1}, \ldots, V_{n-1}\right)$ the sum of polynomials in Theorem 1 (R3) for all $L$ with $|L|=p$. Note that deg $g_{p}\left(R(n, r), V_{1}, \ldots, V_{n-1}\right)=p-1$. We use the notation $\widetilde{\mathbf{y}}$ in the proof of Lemma 6 . We claim the following:

$$
\begin{aligned}
& \sum_{j=0}^{k(n, r)} R(n, r)^{k(n, r)-j} g_{((n+r) / 2)+j}\left(R(n, r), V_{1}, \ldots, V_{n-1}\right) \tilde{y}_{j} \\
& =R(n, r)^{\phi(n, r)+1} .
\end{aligned}
$$

In order to prove (55), we define

$$
\begin{aligned}
& C_{n, r}=\left(\left(\begin{array}{c}
D(n)+1-i \\
e(n, r)-j
\end{array}\right)\right)_{\substack{0 \leq i \leq e(n, r) \\
0 \leq j \leq e(n, r)-1}}, \\
& \widetilde{C}_{n, r}=\left(\left(\begin{array}{c}
D(n)+1-i \\
e(n, r)-j
\end{array}\right)\right)_{\substack{0 \leq i \leq e(n, r) \\
0 \leq j \leq k(n, r)}} .
\end{aligned}
$$

Then the following equality holds:

$$
\widetilde{C}_{n, r}{ }^{t}\left(\widetilde{y}_{0}, \ldots, \widetilde{y}_{k(n, r)}\right)=C_{n, r}{ }^{t} \widetilde{\mathbf{y}}={ }^{t}(1,0, \ldots, 0) .
$$

In fact, the left equality follows from the fact that $\tilde{y}_{i}=0$ for $k(n, r)+1 \leq i \leq e(n, r)-1$. On the other hand, the right equality is proved as follows. Note that we can write $C_{n, r}$ as

$$
C_{n, r}=\left(\begin{array}{c}
\mathbf{c} \\
\widetilde{A}_{e(n, r)}(D(n)-e(n, r))
\end{array}\right),
$$

where $\mathrm{c}$ is the first row of $C_{n, r}$ and $\widetilde{A}_{e(n, r)}(D(n)-e(n, r))$ is the matrix defined from $A_{e(n, r)}(D(n)-e(n, r))$ by shifting the $(i, j)$-element to the $(e(n, r)+1-i, e(n, r)+1-j)$ element. Since ${ }^{t} \mathbf{y} \in \operatorname{Ker} A_{e(n, r)}(D(n)-e(n, r))$, we have ${ }^{t} \widetilde{\mathbf{y}} \in \operatorname{Ker} \widetilde{A}_{e(n, r)}(D(n)-e(n, r))$. Now the right equality of (57) follows from Proposition 4(iii)(b).

Let $\sigma_{i}$ be the $i$ th symmetric polynomial in variables $V_{1}, \ldots, V_{n-1}$. Then we have

$$
g_{((n+r) / 2)+j}=\sum_{i=0}^{e(n, r)}\left(\begin{array}{c}
D(n)+1-i \\
e(n, r)-j
\end{array}\right) R(n, r)^{((n+r) / 2)+j-i-1} \sigma_{i} .
$$

In fact, the binomial coefficient is computed from $\left(\left(\begin{array}{c}n-1 \\ p\end{array}\right)\left(\begin{array}{c}p \\ i\end{array}\right)\right) /\left(\begin{array}{c}n-1 \\ i\end{array}\right)$ for $p=((n+r) / 2)+j$. Now we complete the proof of (55) as follows:

the left hand side of (55)

$$
\begin{gathered}
=\left(R(n, r)^{((n+r) / 2)+k(n, r)-1} \sigma_{0}, \ldots,\right. \\
\left.R(n, r)^{((n+r) / 2)+k(n, r)-e(n, r)-1} \sigma_{e(n, r)}\right) \\
\times \widetilde{C}_{n, r}{ }^{t}\left(\tilde{y}_{0}, \ldots, \widetilde{y}_{k(n, r)}\right) \quad(\text { by }(59)) \\
=\left(R(n, r)^{((n+r) / 2)+k(n, r)-1} \sigma_{0}, \ldots,\right. \\
\left.R(n, r)^{((n+r) / 2)+k(n, r)-e(n, r)-1} \sigma_{e(n, r)}\right)
\end{gathered}
$$

$$
\begin{aligned}
& \times{ }^{t}(1,0, \ldots, 0) \quad(\text { by }(57)) \\
= & R(n, r)^{((n+r) / 2) k(n, r)-1}=R(n, r)^{\phi(n, r)+1} .
\end{aligned}
$$

Hence (55) holds.

Now by Theorem 1 (R3), we have

$$
g_{p}\left(R(n, r), V_{1}, \ldots, V_{n-1}\right)=0
$$

in $H^{p-1}\left(\bar{M}_{n, r} ; \mathbb{Z}_{2}\right)$. Since the left-hand side of (55) vanishes, so does the right-hand side.

Proof of Theorem $A$. We prove the theorem by induction on $n$ and for all $r$.

(I) The case for $n=4$.

Since $\bar{M}_{4,2}=S^{1}$, Theorem A holds.

(II) Fix $n$ and assume that Theorem A holds for all $k$ with $k<n$.

We shall prove Theorem A for $\bar{M}_{n, r}$.

Case (i) $(2 \leq r \leq n-2)$.

We need to consider the cases according to $\left(\begin{array}{c}D(n) \\ e(n, r)\end{array}\right) \equiv 0$ or 1 . Since the case of 1 is proved in Theorem $B$, we may assume that $\left(\begin{array}{c}D(n) \\ e(n, r)\end{array}\right) \equiv 0$. We define an inclusion $i: M_{n-1, r-1} \rightarrow M_{n, r}$ by

$$
i\left(z_{1}, \ldots, z_{n-2}\right)=\left(z_{1}, \ldots, z_{n-2}, 1\right) .
$$

Note that the map $i$ naturally induces a map $\bar{i}: \bar{M}_{n-1, r-1} \rightarrow$ $\bar{M}_{n, r}$ and we have the following diagram of regular 2-covers:

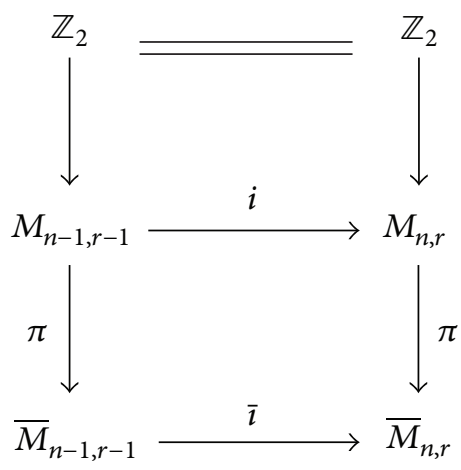

Since $R(n, r)$ is the first Stiefel-Whitney class of the regular 2-cover $\pi: M_{n, r} \rightarrow \bar{M}_{n, r}$, we have

$$
\bar{\imath}^{*}(R(n, r))=R(n-1, r-1) .
$$

By inductive hypothesis, we have in $H^{*}\left(\bar{M}_{n-1, r-1} ; \mathbb{Z}_{2}\right)$ that

$$
R(n-1, n-1)^{\phi(n-1, r-1)} \neq 0 .
$$

Using (64), we have in $H^{*}\left(\bar{M}_{n, r} ; \mathbb{Z}_{2}\right)$ that

$$
R(n, r)^{\phi(n-1, r-1)} \neq 0 .
$$

Using Lemma 6(i), we have $R(n, r)^{\phi(n, r)} \neq 0$. 
On the other hand, we have by Proposition 8 that $R(n, r)^{\phi(n, r)+1}=0$. Hence we have proved that $h(n, r)=$ $\phi(n, r)$.

Case (ii) $(r=1)$.

As in (i), we may assume that $\left(\begin{array}{c}D(n) \\ e(n, r)\end{array}\right) \equiv 0$. Lemma 6(ii) tells us that if $2^{s}+3 \leq n \leq 2^{s+1}-1$, then $\phi(n, 1)=2^{s}-2$. Moreover, Proposition 8 tells us that $R(n, 1)^{2^{s}-1}=0$. Hence it will suffice to prove that $R(n, 1)^{2^{s}-2} \neq 0$ in $H^{*}\left(\bar{M}_{n, 1} ; \mathbb{Z}_{2}\right)$. We define an inclusion $j: M_{2^{s}+1,1} \rightarrow M_{n, 1}$ by

$$
i\left(z_{1}, \ldots, z_{2^{s}}\right)=(z_{1}, \ldots, z_{2^{s}}, \underbrace{1, \ldots, 1}_{d}, \underbrace{-1, \ldots,-1}_{d}) \text {. }
$$

The map induces a map $\bar{\jmath}: \bar{M}_{2^{s}+1,1} \rightarrow \bar{M}_{n, 1}$. Using a similar diagram to (63) and the fact that $R\left(2^{s}+1,1\right)^{2^{s}-2} \neq 0$ in $H^{*}\left(\bar{M}_{2^{s}+1,1} ; \mathbb{Z}_{2}\right)$ (see Theorem B), we have in $H^{*}\left(\bar{M}_{n, 1} ; \mathbb{Z}_{2}\right)$ that $R(n, 1)^{2^{s}-2} \neq 0$.

Proof of Proposition C. Proposition C(i)(a) and(b) follow from Theorem A and Lemma 6(ii), and (i)(c) follows from Theorem B. If we modify Lemma 6(ii) slightly for even $n$, then we can prove Proposition C(ii) similarly.

\section{References}

[1] J. C. Hausmann, "Sur la topologie des bras articules," in Algebraic Topology Poznań, vol. 1474 of Lecture Notes in Mathematics, pp. 146-159, Springer, Berlin, Germany, 1989.

[2] M. Kapovich and J. Millson, "On the moduli space of polygons in the Euclidean plane," Journal of Differential Geometry, vol. 42, no. 2, pp. 430-464, 1995.

[3] K. Walker, Configuration spaces of linkages [Undergraduate thesis], Princeton University, 1985.

[4] J.-C. Hausmann and A. Knutson, "The cohomology ring of polygon spaces," Annales de l'Institut Fourier, vol. 48, no. 1, pp. 281-321, 1998.

[5] M. Farber, J. Hausmann, and D. Schütz, "On the conjecture of Kevin Walker," Journal of Topology and Analysis, vol. 1, no. 1, pp. 65-86, 2009.

[6] Y. Kamiyama, M. Tezuka, and T. Toma, "Homolog y of the configuration spaces of quasi-equilateral polygon linkages," Transactions of the American Mathematical Society, vol. 350, no. 12, pp. 4869-4896, 1998.

[7] H. Hiller, "On the cohomology of real Grassmannians," Transactions of the American Mathematical Society, vol. 257, pp. 521-533, 1980. 


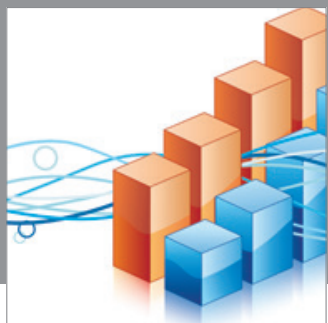

Advances in

Operations Research

mansans

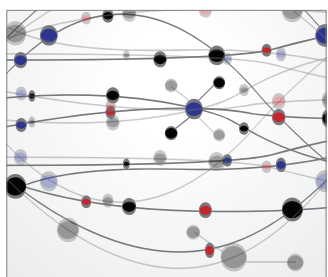

The Scientific World Journal
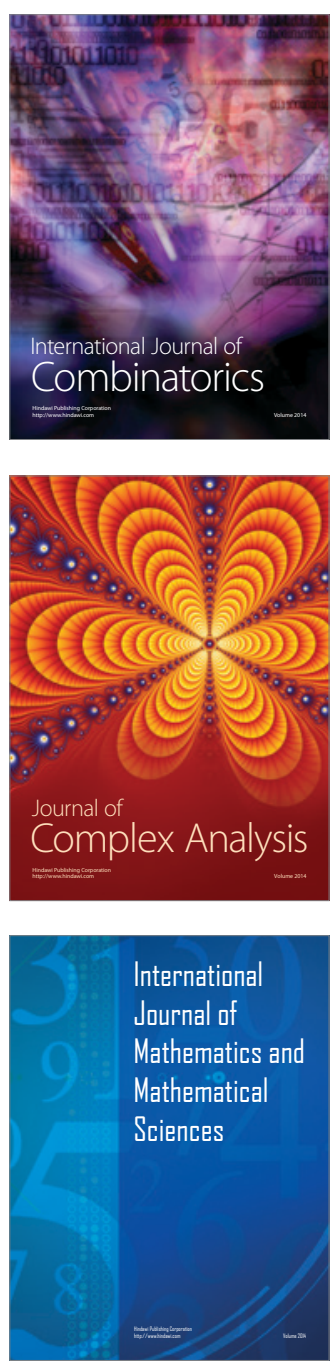
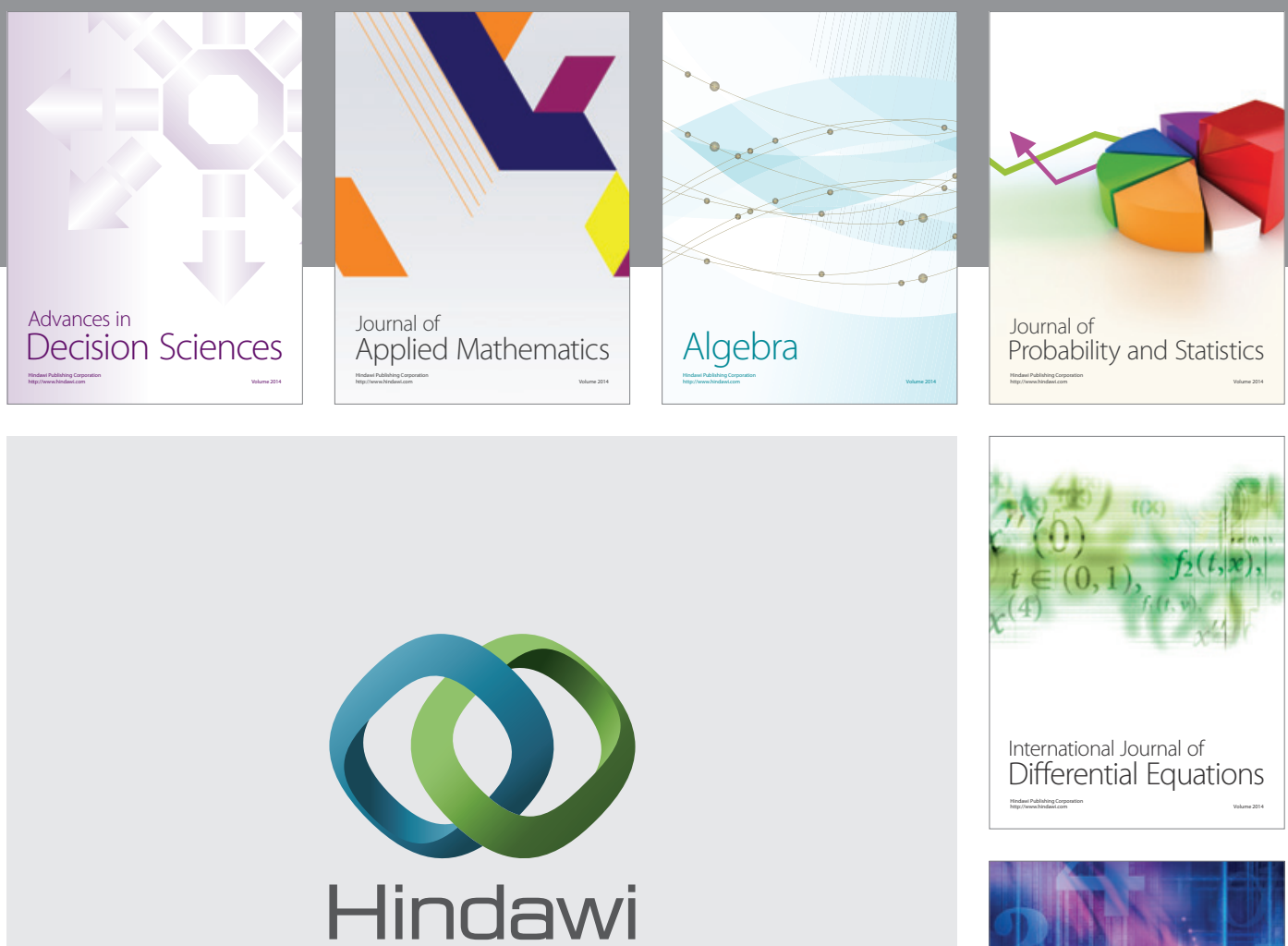

Submit your manuscripts at http://www.hindawi.com
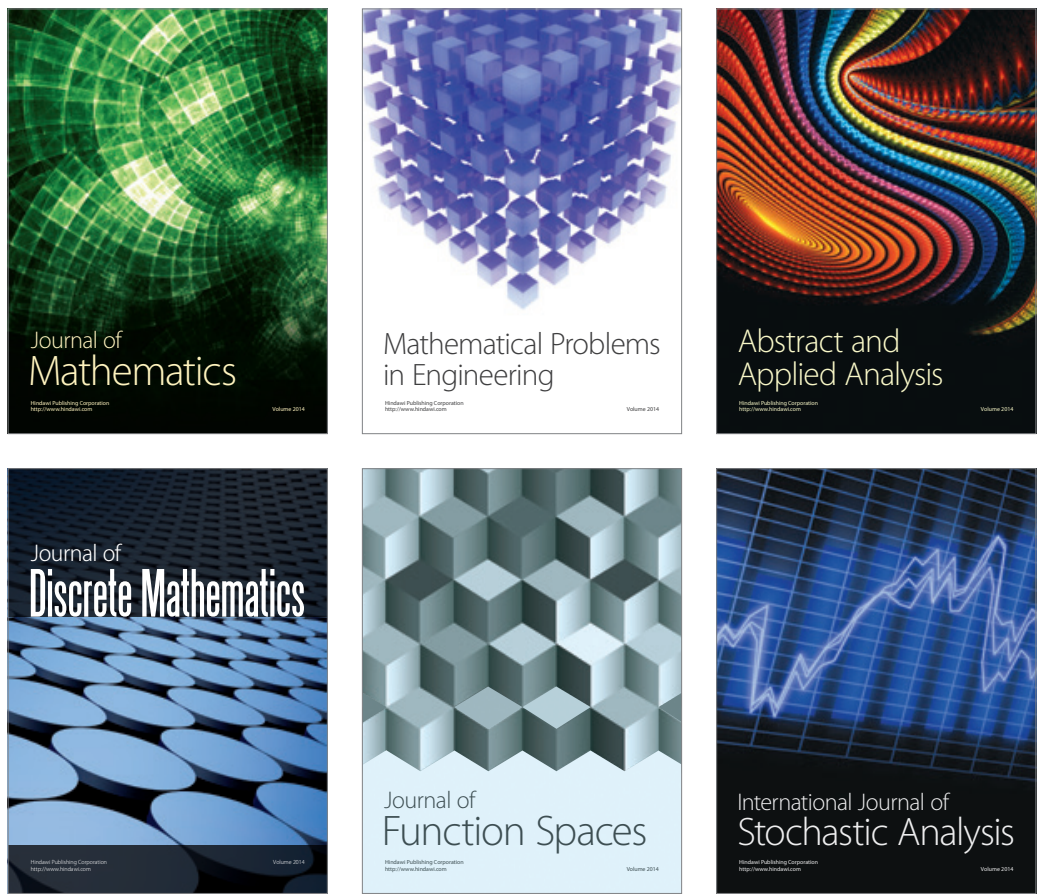

Journal of

Function Spaces

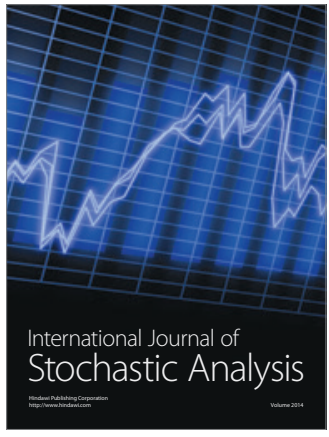

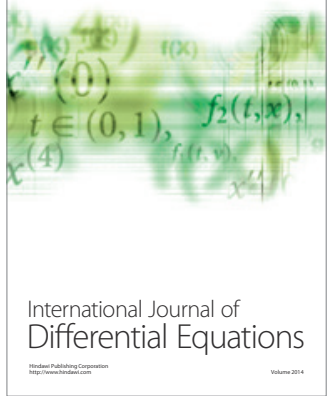
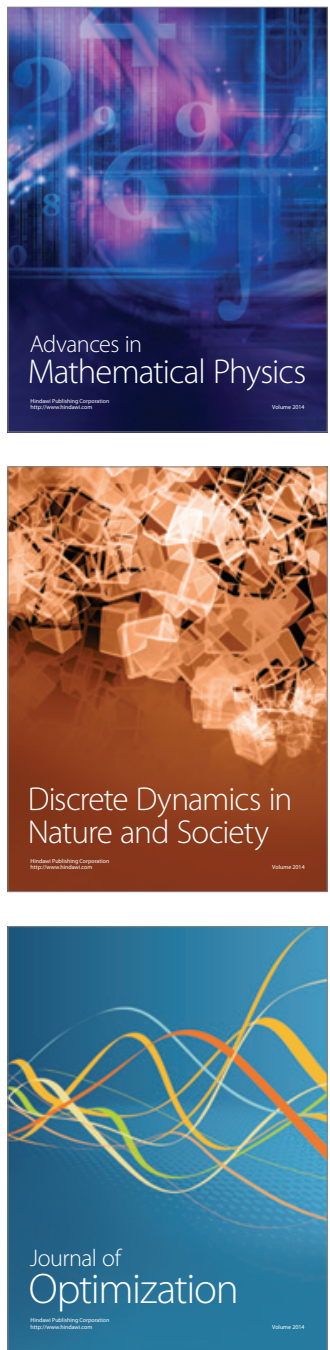